\title{
Gastric compression due to a cystic liver metastasis of vulvar carcinoma diagnosed by endoscopic ultra- sound-guided fine-needle aspiration
}

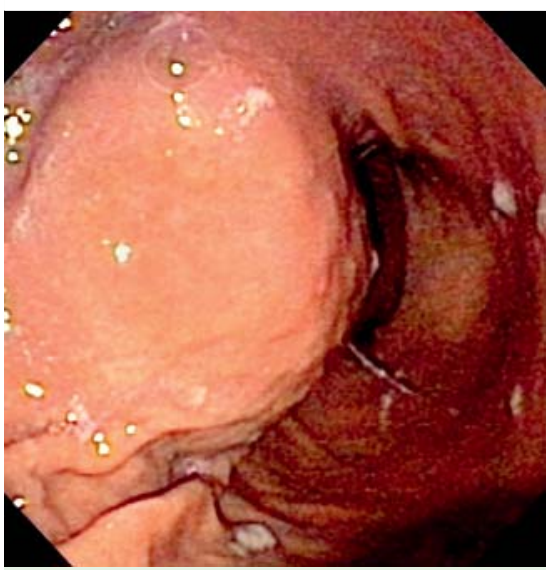

Fig. 1 Endoscopic view of a bulge in the lesser curvature and anterior wall of the proximal gastric body.

A 63-year-old woman who was complaining of epigastric pain and abdominal fullness underwent an upper gastrointestinal endoscopy, which revealed a bulge in the lesser curvature and anterior wall of the gastric body ( Fig.1). A computed tomography (CT) scan and a sectorial endoscopic ultrasound (EUS) using an Olympus GF-UCT140-AL5 (Olympus America Inc., New York, USA) coupled to an ultrasound unit (Aloka Prosound alfa-5 SX) detected a 5 -cm cyst in the caudate lobe of the liver ( Fig.2). A definitive diagnosis was obtained by endoscopic ultrasound-guided fine-needle aspiration (EUS-FNA). Only a single needle pass was performed with a 19-gauge needle (EchoTip Ultra Echo-19; Cook Medical, Winston-Salem, North Carolina, USA), and the content of the cyst $(90 \mathrm{~mL})$ was completely aspirated. A cystic liver metastasis of vulvar carcinoma was confirmed by immunohistochemistry that was consistent with this diagnosis ( Fig.3). The patient subsequently underwent surgery, with complete resection of the lesion.

Vulvar carcinoma is a rare genital malignancy, accounting for less than $1 \%$ of all malignancies in women, and up to $5 \%$ of malignancies of the genital tract [1]. Most of these cancers are squamous cell carcinomas, and surgery is the only chance of cure. Absence of lymph nodes metastases

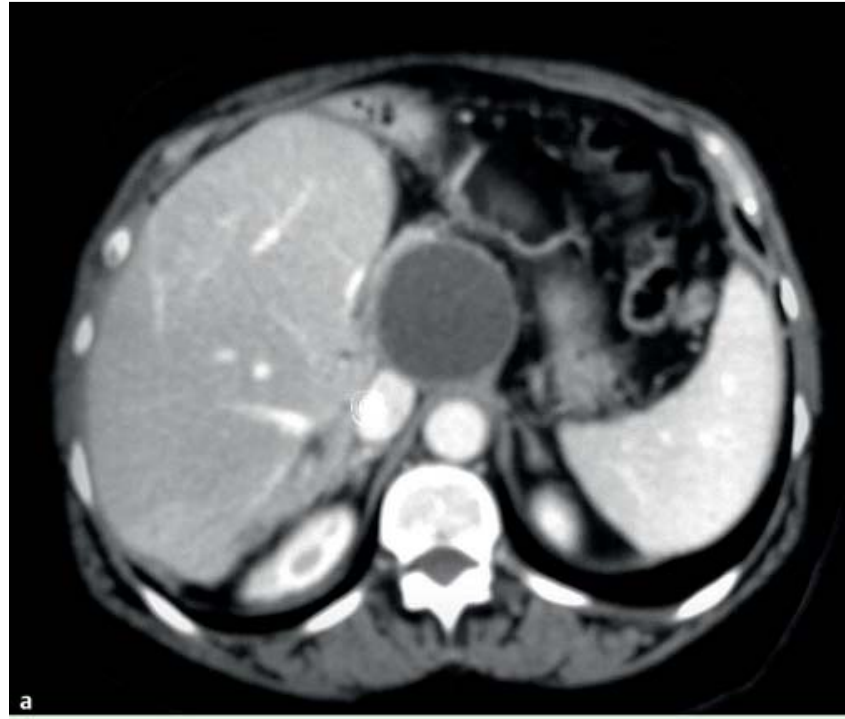

Fig. 2 A cyst in the caudate lobe of the liver shown on: a a computed tomography (CT) scan as a 5-cm single cyst; b linear-array endoscopic ultrasound (EUS), which reveals a single thin-walled cyst measuring $5 \times 4 \mathrm{~cm}$, with well-defined borders and no septation or mural nodules.

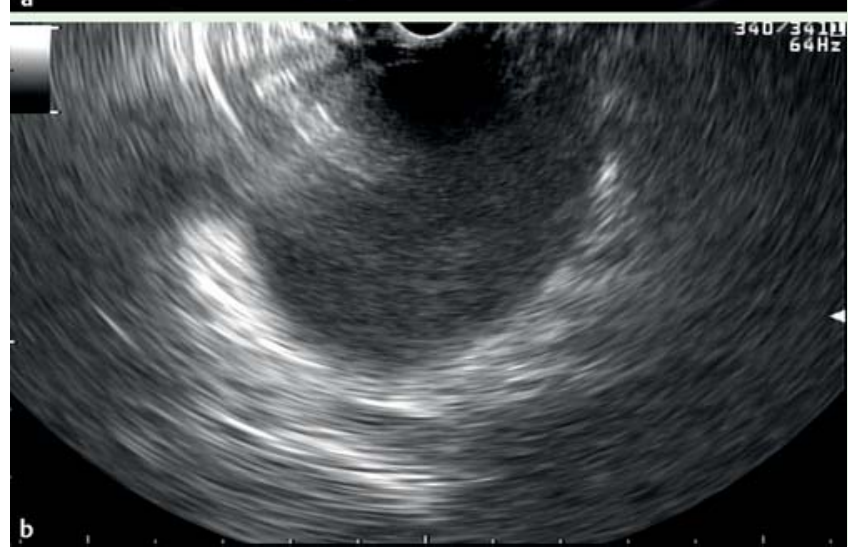

and distant metastases, which are more common in larger and deeper tumors, are prognostic factors for disease-free survival [1]. The disease usually metastasizes to pelvic lymph nodes, with hematogenic metastases to distant organs occurring in less than $10 \%$ of patients with advanced disease. Liver metastasis secondary to vulvar carcinoma is infrequent, with only a few cases having been reported in the literature [2]. Cystic liver metastases are very uncommon. In addition, to the best of our knowledge, this is the first reported case of an EUS-FNA diagnosis of a vulvar cancer that had metastasized to the liver. In this case, a very late cystic liver metastasis occurred after resection of an early vulvar squamous cell carcinoma (pTisNOM0)
13 years previously. EUS imaging alone is not predictive of neoplastic lesions, and these cases can be misdiagnosed as simple cysts. However, EUS is the best option for clarifying the differential diagnosis between extraluminal compression and subepithelial tumor [3], which was the initial finding in this case. EUS-FNA with histological confirmation of liver cysts in women with a previous history of genital tract malignancy is a valuable method for the diagnosis of a cystic liver neoplasm [4].

Endoscopy_UCTN_Code_CCL_1AG_2AB

Competing interests: None 


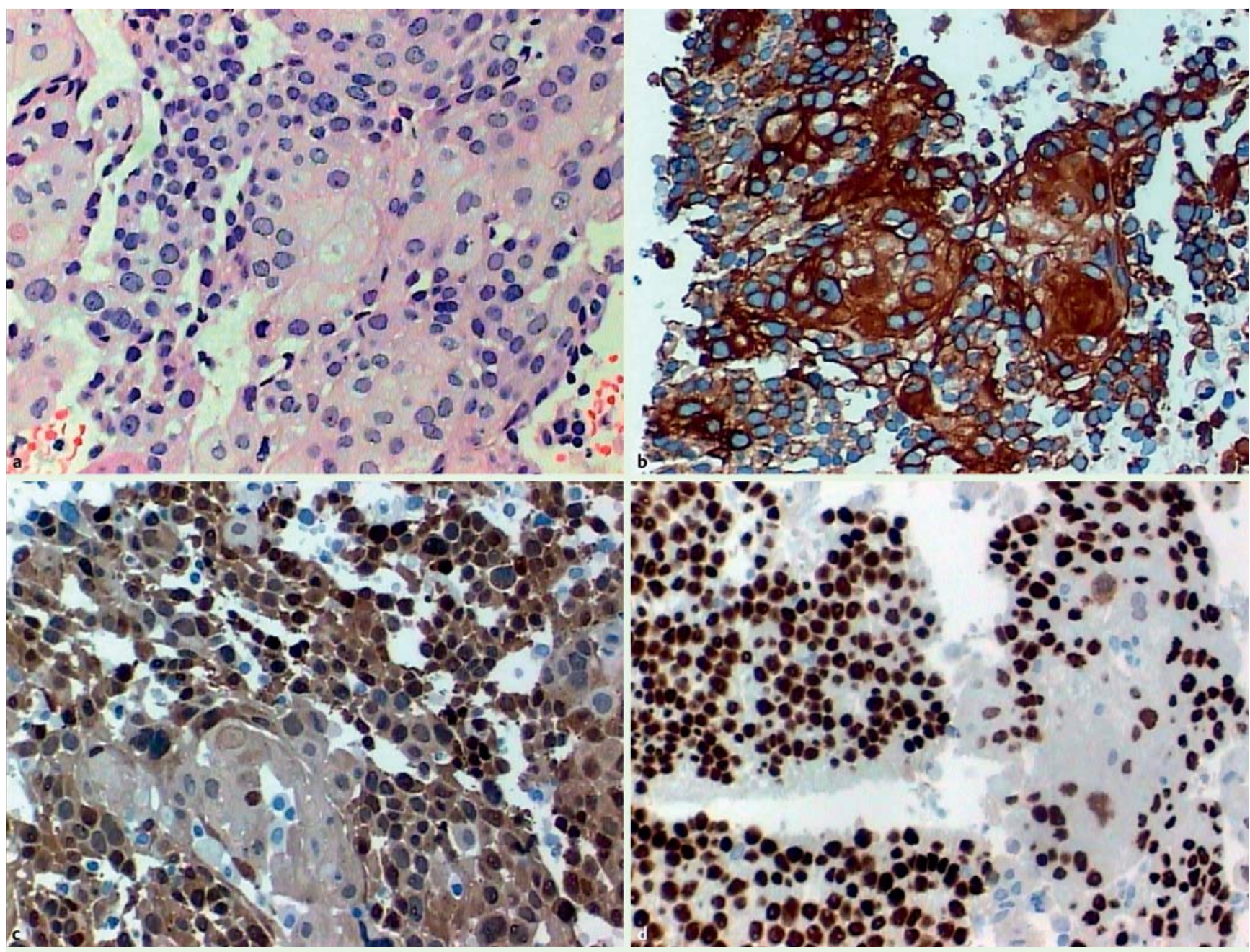

Fig. 3 Histopathologic appearance (original magnification $\times 200$ ) of the material obtained by endoscopic ultrasound-guided fine needle aspiration $(E U S-F N A)$ demonstrating: a a poorly differentiated squamous cell carcinoma composed of markedly pleomorphic cells with eccentric nuclei and prominent nucleoli on hematoxylin and eosin (H\&E) staining; and positive immunohistochemical staining with: b cytokeratin M (clones AE1/AE3); c p63 (clone 7 Jul); and d p16 (clone $6 \mathrm{H} 12$ ), confirming the diagnosis of a liver metastasis of vulvar carcinoma.

César Vivian Lopes ${ }^{1}$, Régis Garcia de Garcia', Gabriel Oliveira dos Santos², Antônio Atalíbio Hartmann², Antônio Nocchi Kalil ${ }^{3}$

${ }^{1}$ Department of Gastroenterology and Digestive Endoscopy, Santa Casa Hospital, Porto Alegre, Brazil

2 Department of Pathology, Santa Casa Hospital, Porto Alegre, Brazil

Department of Surgical Oncology, Santa Casa Hospital, Porto Alegre, Brazil
References

1 Coulter J, Gleeson N. Local and regional recurrence of vulval cancer: management dilemmas. Best Pract Res Clin Obstet Gynaecol 2003; 17: 663-681

2 Woolderink JM, de Bock GH, de Hullu JA et al. Patterns and frequency of recurrences of squamous cell carcinoma of the vulva. Gynecol Oncol 2006; 103: 293-299

3 Rösch T, Kapfer B, Will $U$ et al. Endoscopic ultrasonography. Accuracy of endoscopic ultrasonography in upper gastrointestinal submucosal lesions: a prospective multicenter study. Scand J Gastroenterol 2002; 37: 856-862

4 tenBerge J, Hoffman BJ, Hawes RH et al. EUSguided fine needle aspiration of the liver: indications, yield, and safety based on an international survey of 167 cases. Gastrointest Endosc 2002; 55: 859-862
Bibliography

DOI http://dx.doi.org/

10.1055/s-0034-1365385

Endoscopy 2014; 46: E208-E209

(c) Georg Thieme Verlag KG

Stuttgart · New York

ISSN 0013-726X

Corresponding author

César Vivian Lopes, MD, PhD

Rua Prof. Cristiano Fischer 668/1001

CEP 91410-000 Porto Alegre

RS, Brazil

Fax: +55-51-33388054

drcvlopes@gmail.com 